\title{
New Approach for Ground Water Detection Monitoring at Lined Landfills
}

\author{
by N. Buket Yenigül, Amro M.M. Elfeki, and Cees van den Akker
}

\begin{abstract}
The design of a ground water detection monitoring system at a lined landfill is complicated due to uncertainties in contaminant source characteristics and variability of hydrogeological conditions. Maximizing the likelihood of detecting contaminants and minimizing the contaminated area are the conflicting design objectives. Mostly, a large number of wells may be required to achieve the desired efficiency. However, the cost might be quite high from a practical point of view. Moreover, with the conventional monitoring approach, a widely applied three-well monitoring system (minimum regulatory requirement) is more often inadequate to accomplish these objectives at lined landfills due to the limited capture zone of monitoring wells. Therefore, implementation of a new monitoring approach has been proposed in this study to design a highly efficient, cost-effective, three-well system. In this new approach, the main idea is to increase the interception of contaminant plumes at early stages by broadening the capture zone of monitoring well(s) simply by continuous pumping from the monitoring well(s) with a small pumping rate. A hypothetical problem is presented where a Monte Carlo framework is used to incorporate uncertainties due to subsurface heterogeneity and the leak location. A finite-difference ground water model coupled with a random-walk particle-tracking model simulates a contaminant plume released from the landfill for each Monte Carlo realization. The efficiency and the cost of the three-well monitoring network have been compared for conventional and proposed monitoring approaches $(P M A)$. It has been observed that the efficiency of the monitoring system improves significantly by the application of the $P M A$.
\end{abstract}

\section{Introduction}

The focus of ground water investigation has traditionally been on quantification of this resource, but increasing detections of contaminants during the last decades have shifted this focus toward assessment and protection of ground water quality. A growing awareness of the environmental effects of waste disposal presents a great challenge to those who are in charge of ground water protection. Despite the nowadays design criteria and techniques, which aim to minimize the chance of leakage, the risk of contamination cannot be completely eliminated. Therefore, regulatory agencies require ground water monitoring programs at solid waste landfills, hazardous waste sites, and other sites where potential release of chemicals to the subsurface is a concern in order to have information about contaminant movement and the concentration distribution in the event of a release.

\section{Problem Definition}

The purpose of detection monitoring is early detection of a release to ground water, should one occur, based on comparison of downgradient well data to background data for a limited number of water quality parameters. However, in practice, design of an efficient detection monitoring system is often difficult and complicated due to the uncertainties in specific characteristics of the source of contamination and in the spatial variability of the hydrogeological characteristics, which make ground water flow and contaminant paths hard to predict. Locations, depth, and number of monitoring wells, chemical characteristics of contaminants, and sampling are the other significant parameters that affect the efficiency of a monitoring system. For detection monitoring, regulations require at least one background well (hydraulically upgradient from a potential source) and three downgradient wells (U.S. EPA 1986; ECC 1999). The position, number (more than the minimum requirement), and depth of the monitoring wells are proposed by the landfill owners or operators and by local authorities. The conventional monitoring program suggested by regulatory agencies requires the monitoring of ground water 
quarterly, biannually, or annually, depending on the type of waste, size and design of landfill, and aquifer material for 30 years of postclosure monitoring duration. In most cases, a quarterly monitoring is undertaken; annual monitoring is undertaken mostly for small landfills located in remote places far away from any ground water-use source. There is no recognition of uncertainty in regulations conversely to reality.

Certain aspects of this problem have been discussed in the literature. Massmann and Freeze (1987) presented a risk cost-benefit analysis for a waste management facility from the perspective of the owner/operator to make design decisions for a facility. Rouhani and Hall (1988) investigated the significance of a sampling program in network design by using a method based on variance reduction analysis, media ranking, and risk, but did not consider uncertainty. Meyer et al. (1994) used a multiobjective stochastic optimization approach to determine the two-dimensional location of monitoring wells, incorporating uncertainty in hydraulic conductivity and source location through Monte Carlo simulations. Storck et al. (1997) extended this model to three dimensions incorporating local dispersion. Their approach appears to be very computationally intensive due to solving the optimization module at every iteration. Mahar and Datta (1997) used an optimization approach to design a ground water quality monitoring network and to identify the source of contamination. They did not address the effects of parameter uncertainty in their work. Hudak (2002) presented a deterministic graphical approach to evaluate detection capabilities of perpendicular and equidistant ground water monitoring networks in aquifers dominated by intergranular porosity.

In previous studies, it has been observed that mostly more than three wells are required for an optimal system that will enable satisfactory high detection probability while minimizing the expected contaminated area of the aquifer and/or the total cost of the system. Similarly, the detection monitoring problem is formulated with the main objectives of maximization of the probability of detecting contaminants, minimization of average contaminated area, and minimization of total cost of the system. However, different from earlier studies, this study proposes a new ground water monitoring approach, namely, pumping continuously from the monitoring well(s) with a quite low discharge rate, to improve the efficiency of the widely applied common practice of three downgradient wells (the minimum regulatory requirement). To the best of the authors' knowledge, this approach has not been considered before in monitoring network design.

In the conventional monitoring approach (CMA), the water level of each monitoring well is purged by removing four well volumes (internal radius of the well $x$ the height of the water column in the well) of water using a bailer or a pump before taking a sample to monitor the ground water quality. Although it is technically feasible to maximize the likelihood of detecting contaminant plumes with such $C M A$, the cost might be quite high to be practical due to the necessity of a large number of wells to implement the desired high performance, particularly at lined landfill sites. The initial leakage from a lined landfill will be from point sources such as holes, tears, and imperfections in the liner systems. These point sources will produce ground water plumes that move as fairly narrow especially in low-dispersive medium, where the lateral spreading is relatively limited in the distance between the landfill and the point of compliance for ground water monitoring. Moreover, in practice the budget constraint allows the use of a very limited number of wells in monitoring systems and more often the monitoring systems are composed of three downgradient wells, which fulfils the minimum regulatory requirements. Therefore, considering the nature of incipient leakage from the lined landfills and the limited capture zone of conventional monitoring wells, which are widely spaced, it is very likely that the current practice of ground water monitoring systems at lined landfills instills a false sense of security and is of little use in protecting ground water resources from pollution by leachates.

However, in the new proposed monitoring approach (PMA), the capture zone of monitoring wells broadens due to the pumping from the wells. Therefore, the chance of intercepting more contaminated plumes at early times is expected to increase. Thus, the main objective of this study is to evaluate the influence of the new PMA on the efficiency of common monitoring systems and to compare the analysis results with those of the CMA in terms of detection probability, average contaminated area, and cost, while incorporating uncertainties due to hydrogeological and contaminant source characteristics.

\section{Model Framework}

A simulation-based model (adapted from Elfeki 1996) coupled with a two-dimensional, finite-difference flow model and a random-walk, particle-tracking model is used to determine the optimal monitoring system, which enables the highest detection probability and the minimum average contaminated area under uncertainty. A Monte Carlo approach is used to generate a large number of equally likely contaminant plumes resulting from the failure of the lined landfill. A single Monte Carlo realization consists of (1) generation of random hydraulic conductivity field with mean, variance, and exponential autocorrelation function and a random leak location along the downgradient edge of the lined landfill; (2) solution of steady-state ground water flow model to obtain the velocity field; and (3) a randomwalk transport model to determine the concentration field of the contaminant plume (for further information about the model and the sensitivity analysis for the various model parameters, the reader may refer to Yenigül et al. 2005). The outcome of each realization is composed of two items. The first one is a binary variable representing detection (i.e., equals 1) or no detection (i.e., equals zero) of a contaminant plume at a given monitoring well location by checking whether the concentration value at that well exceeds a given threshold concentration. The second one is a variable representing the expected size of the plume at the detection time $\left(A_{\mathrm{d}}\right)$ or at the end of the monitoring period if it is not detected $\left(A_{\mathrm{f}}\right)$. This loop is repeated until the total number of simulation runs is reached. Once all the runs have been 
completed, the detection probability of a system, $P_{\mathrm{d}}$, is estimated as the ratio of the number of simulation runs in which the contaminants are detected to the total number of simulation runs. Finally, the probability of failure of a system to detect the plume, $P_{\mathrm{f}}$, is equal to $\left(1-P_{\mathrm{d}}\right)$. The average contaminated area, $A_{\mathrm{av}}$, is equal to the mean value of expected plume sizes $\left(A_{\mathrm{d}}\right.$ or $\left.A_{\mathrm{f}}\right)$ estimated from the Monte Carlo simulation data.

\section{Hypothetical Problem}

The numerical experiments are carried out using a model of a generic landfill facility and ground water system; however, the dimensions of the model domain and the parameters that have been used are chosen to reflect conditions of typical solid waste landfills. The model domain is defined by $0 \leqslant x \leqslant 500 \mathrm{~m}$ and $0 \leqslant y \leqslant 400 \mathrm{~m}$ (Figure 1). The model is discretized with grid cells of 2 by $2 \mathrm{~m}$ in both $x$ and $y$ directions. In general, the higher the discretization level of flow and transport problems, the better the subsequent solution of flow and transport equations. However, the higher the discretization level, the greater the computational effort required. Therefore, a balance must be found between the level of discretization and the computational expense. Ababou et al. (1989) suggested, as a rule of thumb, the following ratio between the grid cell size $(\Delta x)$ and correlation length $(\lambda)$ :

$$
\frac{\Delta x}{\lambda} \leqslant \frac{1}{\left(1+\sigma_{Y}^{2}\right)}
$$

Bellin et al. (1992) found that a ratio of $\lambda / \Delta x=4$ in the range of $\sigma_{Y}^{2} \leqslant 1.6$ provided satisfactory accuracy and convergence of computations. The principal ratio applied in this paper is $\lambda / \Delta x=7.5$, and it also satisfies the ratio suggested by Ababou et al. (1989). Therefore, the discretization level is considered to be sufficient for all cases tested and presented in the following.

A hypothetical lined landfill is located at $20 \leqslant x \leqslant 50 \mathrm{~m}$ and $140 \leqslant y \leqslant 260 \mathrm{~m}$ in the model domain. For $C M A, 48$

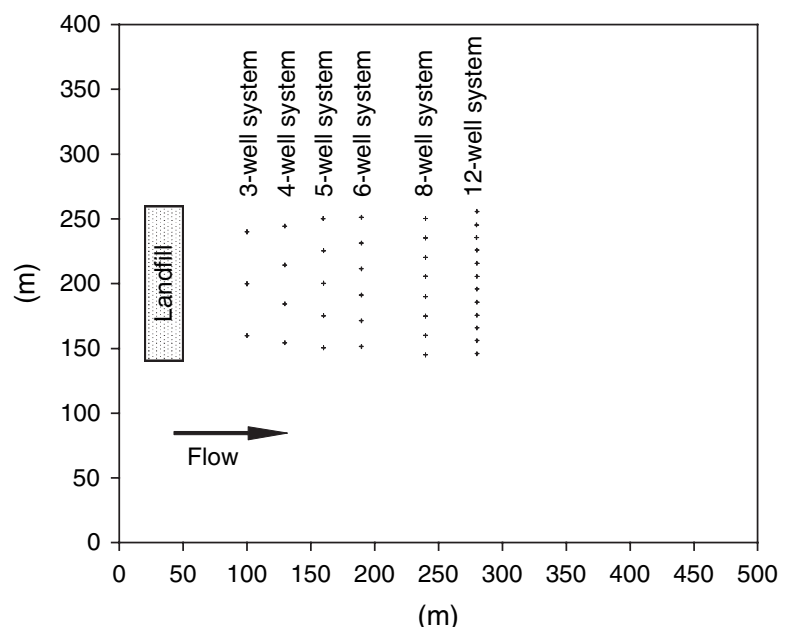

Figure 1. Plan view of the hypothetical problem used in numerical experiments. potential single-row monitoring system alternatives consisting of $3,4,5,6,8$, and 12 wells are located from 20 to $220 \mathrm{~m}$ downgradient of the landfill, with a distance of $30 \mathrm{~m}$ apart. For the PMA, eight monitoring systems of three wells are located also from 20 to $220 \mathrm{~m}$ downgradient of the landfill, with a distance of $30 \mathrm{~m}$ apart. For each alternative, continuous pumping from one well (the well in the middle) is considered. Under the condition of convergence in terms of the number of the Monte Carlo simulations and number of the particles used in the particle-tracking model, the choice of the well, in which the pumping occurs, would have negligible, even no, impact on detection performance of single-row monitoring systems as long as the monitoring wells are evenly spaced and located at a distance equal to half of the well spacing from the top and bottom boundaries of the landfill in order to prevent the boundary effect on detection probability of monitoring wells. Because the wells located at the boundaries will be limited to plumes originating from the leaks at the boundaries or at distances that are very close to the boundaries, this may result in inaccurate detection probability calculations for these wells.

Four different pumping rates, namely, 25, 50, 100, and $1251 /$ day have been used to investigate the influence of pumping rate on the efficiency and the optimality of the monitoring systems. The monitoring system alternatives considered are composed of a line of wells parallel to the $y$ axis, extending the length of the source of contamination.

\section{Parameter Values Used in Flow Model}

The boundary conditions for the ground water flow are zero flux at $y=0 \mathrm{~m}$ (bottom boundary) and $y=400 \mathrm{~m}$ (top boundary) and constant head along the left and the right boundaries. The head values at $x=0 \mathrm{~m}$ and $x=500 \mathrm{~m}$ were chosen to result in a macroscopically constant hydraulic gradient of 0.001 . Porosity equals to 0.25 . Uncertainties due to contaminant source location and subsurface heterogeneity are incorporated in the model. In this study, subsurface heterogeneity is reflected by the spatial variability of the hydraulic conductivity. Hence, hydraulic conductivity is treated as a random space function. The natural logarithm of the isotropic hydraulic conductivity $[Y=\ln (K)]$ is modeled as a stationary Gaussian field with a given mean, variance, and correlation length (e.g., Gelhar 1986). A geometric mean hydraulic conductivity of $2.23 \mathrm{~m} / \mathrm{d}$ is considered, and the variance of $Y$ is set at $\sigma_{Y}^{2}=0$ for homogeneous and at $\sigma_{Y}^{2}=0.5$ for heterogeneous medium. The isotropic covariance of $Y$ is chosen to be of exponential form with a correlation length, $\lambda=15 \mathrm{~m}$.

\section{Parameter Values Used in Random-Walk Particle-Tracking Model}

For the transport model, a condition of a zero dispersive flux is imposed on the top and bottom boundaries, and the initial background concentration in the model domain is set to zero. Since the flow direction is aligned with the $x$ axis, the only source dimension that is treated as a random variable is the position along the $y$ axis. The area of potential leak locations is the downgradient edge of the landfill (Figure 1). The contaminant leak is assumed to be 
a point source, as it would result in a plume, which is most difficult to detect, and the source location is drawn from a uniform probability distribution between $y$ coordinates of $140 \leqslant y \leqslant 260 \mathrm{~m}$ for each Monte Carlo run. Dispersion is incorporated in the model by introducing microscale longitudinal $\left(\alpha_{\mathrm{L}}\right)$ and transverse $\left(\alpha_{\mathrm{T}}\right)$ dispersivities.

The ratio between $\alpha_{\mathrm{L}}$ and $\alpha_{\mathrm{T}}$ is assumed to be 10 (Bear 1972). $\alpha_{\mathrm{L}}$ is set to 0.1 and $0.3 \mathrm{~m}$ to examine the influence of dispersivity. The simulation procedure assumes that the source is continuous and provides a constant mass rate of $1 \mathrm{mg} / \mathrm{L} / \mathrm{d}$. The threshold concentration (detection limit) at which detection occurs is set at $0.5 \%$ of the initial source concentration. Cadmium, benzene, carbon tetrachloride, and 1,1,2-trichloroethane are the most common contaminant types released to ground water mainly via leaks from landfills, and the EPA (U.S. EPA 1986) defines the maximum contaminant level of $0.005 \mathrm{mg} / \mathrm{L}$ for these contaminants in drinking water. Hence, considering a $1 \mathrm{mg} / \mathrm{L}$ initial source of one of these contaminants, the threshold concentration corresponds to $0.005 \mathrm{mg} / \mathrm{L}$, which is equal to the EPA public health risk level for drinking water. Moreover, the number of particles used throughout the example is 2000, and $0.05 \mathrm{mg} / \mathrm{L}$ of a threshold value corresponds to 40 particles, which is a sufficient number for determination of concentration in one grid cell or namely in this study the concentration in a monitoring well (Kinzelbach 1986). Contaminants are assumed to be conservative and to be completely mixed over the depth of the aquifer, which is presumed to be $50 \mathrm{~m}$ in the example problem. A monitoring period of 30 years is considered while monitoring is assumed to be carried out each quarter of a year.

\section{Discussion of Results}

To find answers to the questions "what is the best monitoring system that optimally meets the desired goals, namely, maximizing the likelihood of detecting contaminants under pertinent uncertainties?" and "how to improve the efficiency of widely applied common practice that fulfils the minimum regulatory requirement, namely a three-well system?" and consequently in order to solve the detection monitoring problem, 1000 Monte Carlo simulations are carried out for different subsurface conditions considering both $C M A$ and PMA. The results of the analysis are discussed in the following subsections.

\section{Conventional Monitoring Approach}

\section{Reliability Evaluation}

The reliability of a ground water monitoring system is measured by the probability of detection $P_{\mathrm{d}}$. Figure 2 presents the reliability of monitoring systems at different distances from the source for homogeneous and heterogeneous media. Each data point represents one monitoring system evaluated based on the CMA. Both in homogeneous and heterogeneous media, there is an increase in reliability with distance from the source. Since plumes begin with a small size and spread out as they migrate away from the source, detection of a plume is difficult at close distances
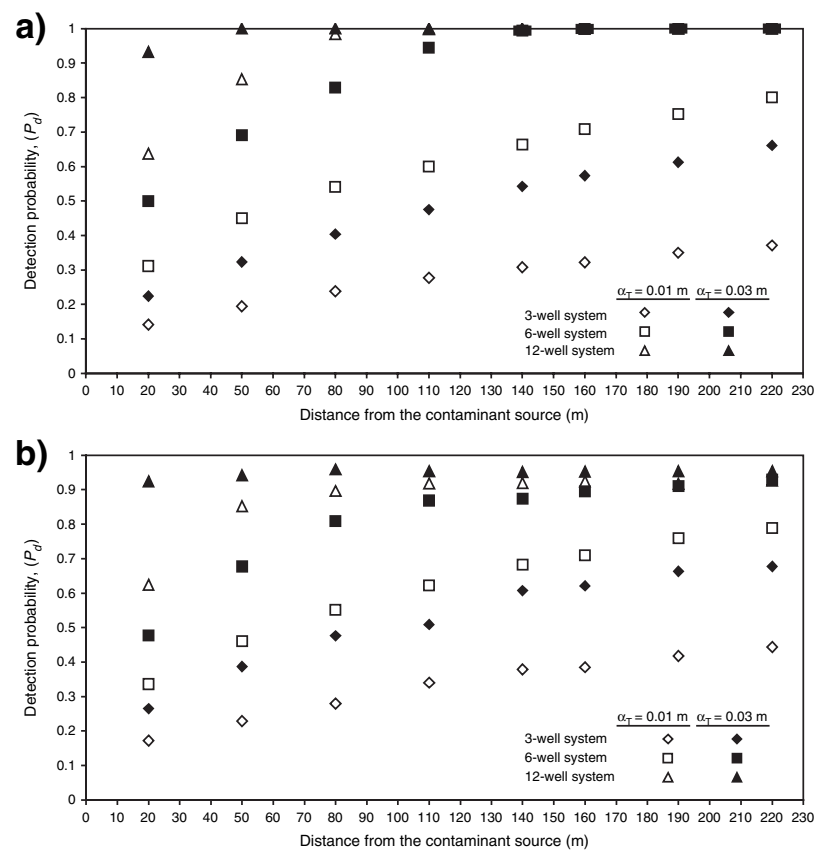

Figure 2. System reliability as a function of distance from the source for selected monitoring systems for CMA: (a) homogeneous medium and (b) heterogeneous medium.

and becomes easier as the plume expands with time and distance from the source. Hence, there is a greater chance of detecting plumes for the systems composed of few wells, when they are placed away from the source. Moreover, as the dispersivity of the medium increases, the detection probability of a given monitoring system increases since dispersivity is the parameter that controls the spreading of the plume (e.g., Meyer et al. 1994; Storck et al. 1997; Yenigül et al. 2005). The higher the dispersivity of the medium, the wider the plume gets as it moves further away from the source. On the other hand, $P_{\mathrm{d}}$ of the monitoring systems are lower in heterogeneous medium since it will be more difficult to detect the contaminant plumes due to their irregular shapes and the uncertainty in the direction that they travel.

$P_{\mathrm{d}}$ of a monitoring system at a given distance increases as the number of wells in the system increases. However, it is important to note that additional wells would not be cost effective for improving detection, particularly in a case where the reliability of the systems is $100 \%$ regardless of the distance from the sources for a certain number of wells. However, for the site dimensions and distance from the source analyzed, the common practice of three wells does not reach $100 \%$ reliability.

\section{Average Contaminated Area}

When a given system detects a contaminant plume, an associated contaminated area is obtained $\left(A_{\mathrm{d}}\right)$. When the monitoring system fails to detect the contaminant plume, the contaminated area at the end of the monitoring period (in this case 30 years) is estimated $\left(A_{\mathrm{f}}\right)$. However, the plume size, either detected or not, varies from one realization to another due to the variability in the source location 
and hydrogeologic characteristics. Therefore, rather than producing a single plume size, a range of plume sizes determined for each monitoring system and the average contaminated area, $A_{\mathrm{av}}$, is equal to the mean value of expected plume sizes (either detected, $A_{\mathrm{d}}$, or not, $A_{\mathrm{f}}$ ) estimated from the Monte Carlo realizations. Both the detection probability of a system and the contaminant area at detection increase as the distance from the source increases, whereas the contaminated area associated with no detection remains basically constant with respect to distance from the source. Therefore, the difference between the size of detected and undetected plumes reduces as the contaminant plumes move away from the source. This will result in the curves presented in Figure 3. The average contaminated area, $A_{\mathrm{av}}$, decreases toward a minimum value up to certain distance and then again increases as distance from the source increases. The distance where the minimum contaminated area occurs is the optimal location where a given monitoring system has to be placed in order to maximize the detection probability while minimizing the contaminated area. $A_{\mathrm{av}}$ increases as the dispersivity of medium increases. As was mentioned previously, this is due to fact that transverse dispersivity is the controlling factor for the spreading and widening of the contaminant plumes. On the other hand, $A_{\mathrm{av}}$ is larger in heterogeneous medium since it is signified by the plumes that are irregular in shape and are more likely to go undetected due to variability in the flow field. Furthermore, the average contaminated area decreases as the number of wells in the monitoring system increases. This is because the detection probability increases as the number of wells in the system increases and consequently $A_{\text {av }}$ decreases since the expected area given no detection is larger than the area given detection. However, when $100 \%$ reliability is achieved by
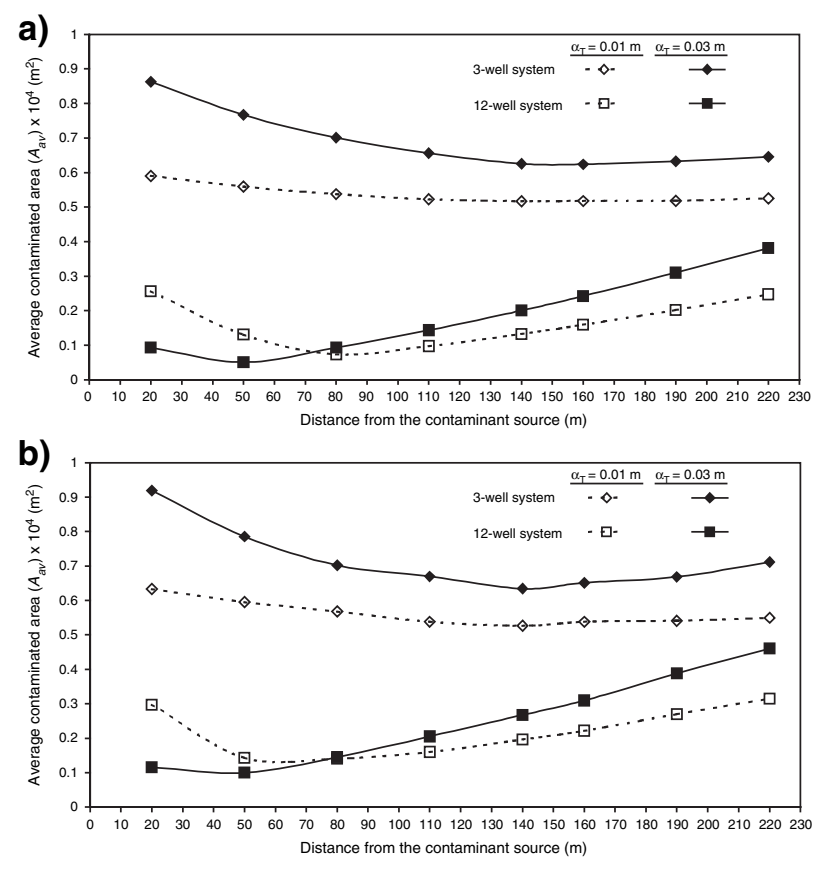

Figure 3. Average contaminated area as a function of distance from the source for selected monitoring systems for $C M A$ : (a) homogeneous medium and (b) heterogeneous medium. a given monitoring system, the addition of wells will not affect the expected extent of contamination as well.

\section{Proposed Monitoring Approach}

\section{Reliability Evaluation and Average Contaminated Area}

The relation between the reliability of the monitoring system, $P_{\mathrm{d}}$, and the distance from the source is the same as it was observed in the results based on the conventional approach (Figures 4 and 5). Hence, $P_{\mathrm{d}}$ of a three-well monitoring system increases as the distance from the source increases, and $A_{\mathrm{av}}$ decreases toward a minimum value up to certain distance and then again increases as distance from the source increases. The distance where the minimum contaminated area occurs is the optimal location where a given monitoring system has to be placed in order to maximize $P_{\mathrm{d}}$ and minimize $A_{\mathrm{av}}$. The quantitative difference in the results of the analysis based on the two approaches will be presented in detail in the Comparison between the $C M A$ and PMA section.

\section{Influence of Pumping Rate}

The influence of the pumping rate on the efficiency of a three-well monitoring system is presented in Figure 6. Both in homogeneous and heterogeneous media, $P_{\mathrm{d}}$ of a three-well system increases as the pumping rate increases. This is due to the fact that the higher the pumping rate, the broader the capture zone of the monitoring well. Therefore, the chance of intercepting contaminated plumes increases as the pumping rate increases and accordingly $A_{\text {av }}$ will decrease. However, the increase in the pumping rate will not make any further improvement once $100 \%$ reliability is achieved at a certain pumping rate.

\section{Comparison between the $C M A$ and $P M A$}

The overall efficiency of a three-well monitoring system improves enormously by application of the PMA. Figures 7 and 8 present $P_{\mathrm{d}}$ as a function of the number of wells in a monitoring system for different subsurface conditions. For a pumping rate of $1001 /$ day, the $P M A$ gives an estimated maximum $P_{\mathrm{d}}$ value of the widely used three-well monitoring system that equals 0.98 in a homogeneous medium and 0.87 in heterogeneous medium even for low dispersivity values $\left(\alpha_{\mathrm{L}}=0.1 \mathrm{~m}, \alpha_{\mathrm{T}}=0.01 \mathrm{~m}\right)$. Under the

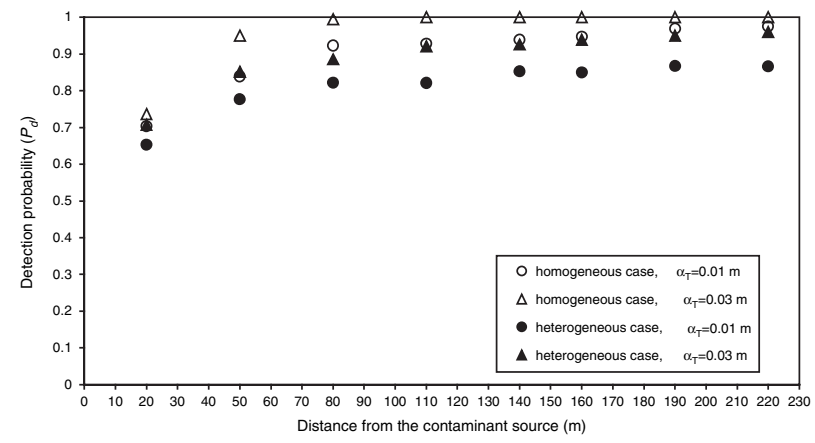

Figure 4. System reliability as a function of distance from the source for a three-well monitoring system for the PMA (pumping rate is $100 \mathrm{~L} / \mathrm{d}$ ). 


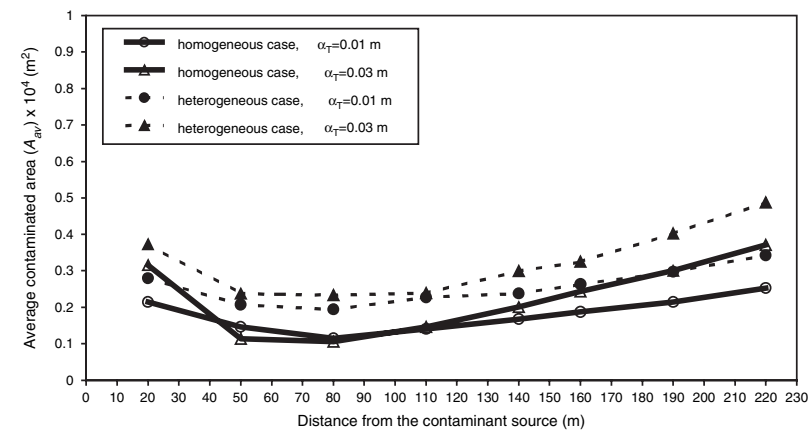

Figure 5. Average contaminated area as a function of distance from the source for a three-well monitoring system for the $P M A$ (pumping rate is $100 \mathrm{~L} / \mathrm{d}$ ).

same circumstances the $C M A$ gives a maximum $P_{\mathrm{d}}$, which does not exceed 0.40 . The efficiency of an optimal threewell system improves by a factor of $\sim 2.3$ (more than $100 \%$ ) by simply pumping with a quite small rate (100 1/day). On the other hand, the maximum $P_{\mathrm{d}}$ values estimated based on the $P M A$ can be achieved by the $C M A$ if 8 or 12 monitoring wells are used, for low and high-dispersive medium, respectively. The detection probability of an optimal threewell monitoring system in the least favorable subsurface conditions (heterogeneous low-dispersive medium) improves by a factor of 2.2 when the PMA is applied. The CMA can achieve this level of reliability by a 12-well monitoring system. Furthermore, the analysis showed that when pumping rate is increased to $125 \mathrm{l} /$ day, the detection probability of the three-well system reached even up to $100 \%$ for all test cases (Figure 6).

The $P M A$ leads to a significant improvement in the average contaminated area regardless of heterogeneity and
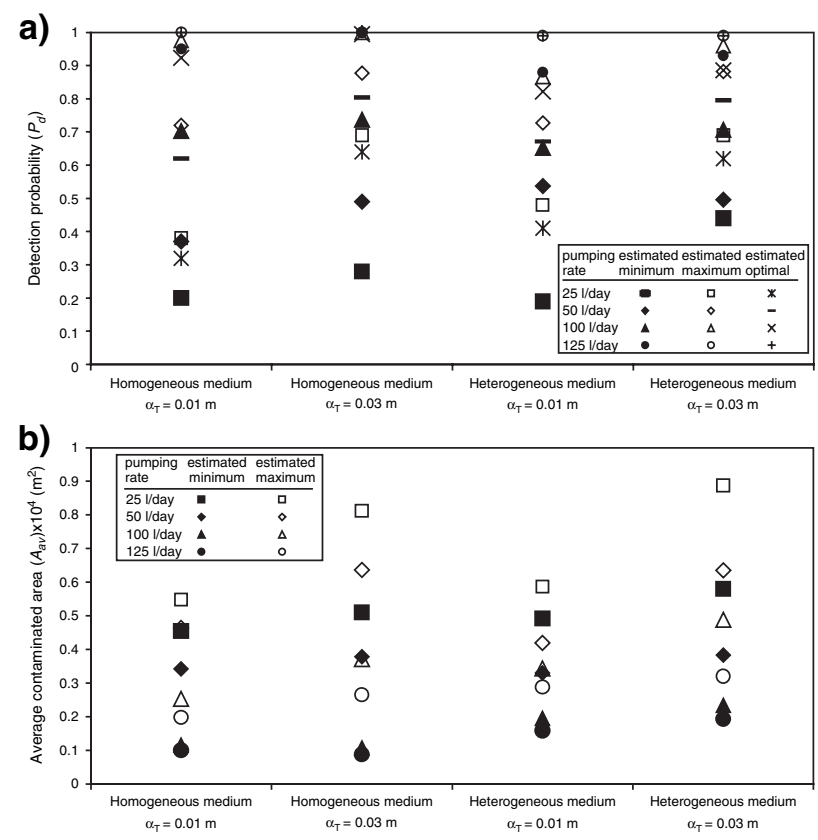

Figure 6. Influence of the pumping rate on (a) detection probability of a three-well system and (b) average contaminated area.
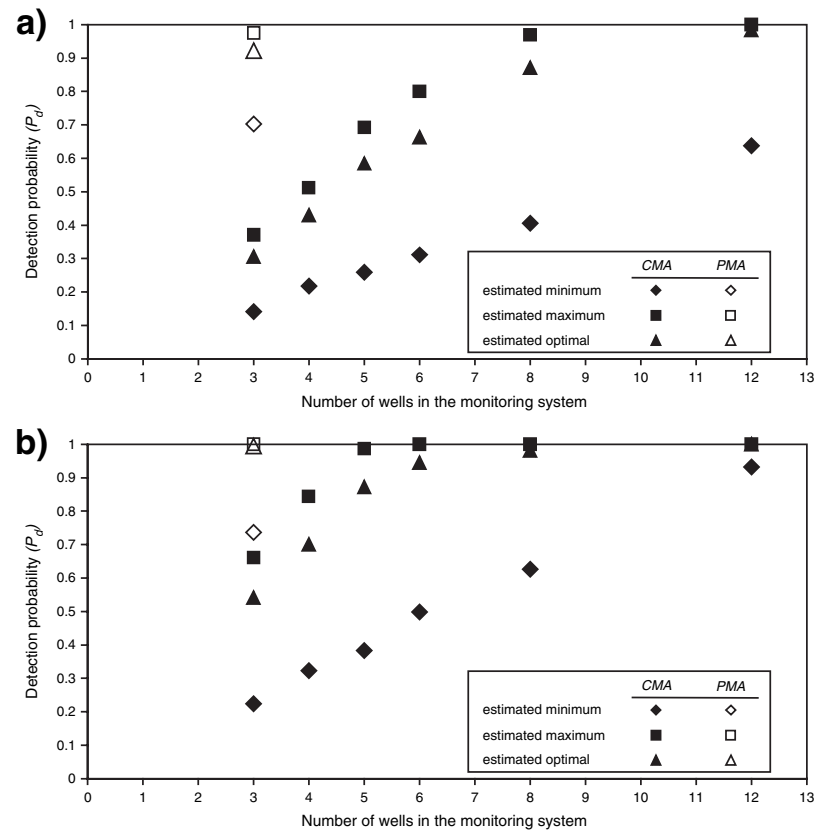

Figure 7. Comparison of the $C M A$ and the $P M A$ (pumping rate $=100 \mathrm{~L} / \mathrm{d}$ ) in terms of reliability in homogeneous medium: (a) transverse dispersivity, $\alpha_{\mathrm{T}}=0.01 \mathrm{~m}$ and (b) transverse dispersivity, $\alpha_{\mathrm{T}}=0.03 \mathrm{~m}$.

dispersivity of medium as well (Figures 9 and 10). For instance, the maximum and minimum contaminated area values associated with a three-well system for the $C M A$ are estimated to be 9190 and $6340 \mathrm{~m}^{2}$ in a heterogeneous highly dispersive medium. For the same monitoring system, when the PMA is applied, the maximum contaminated area is reduced to $4870 \mathrm{~m}^{2}$ and the minimum contaminated
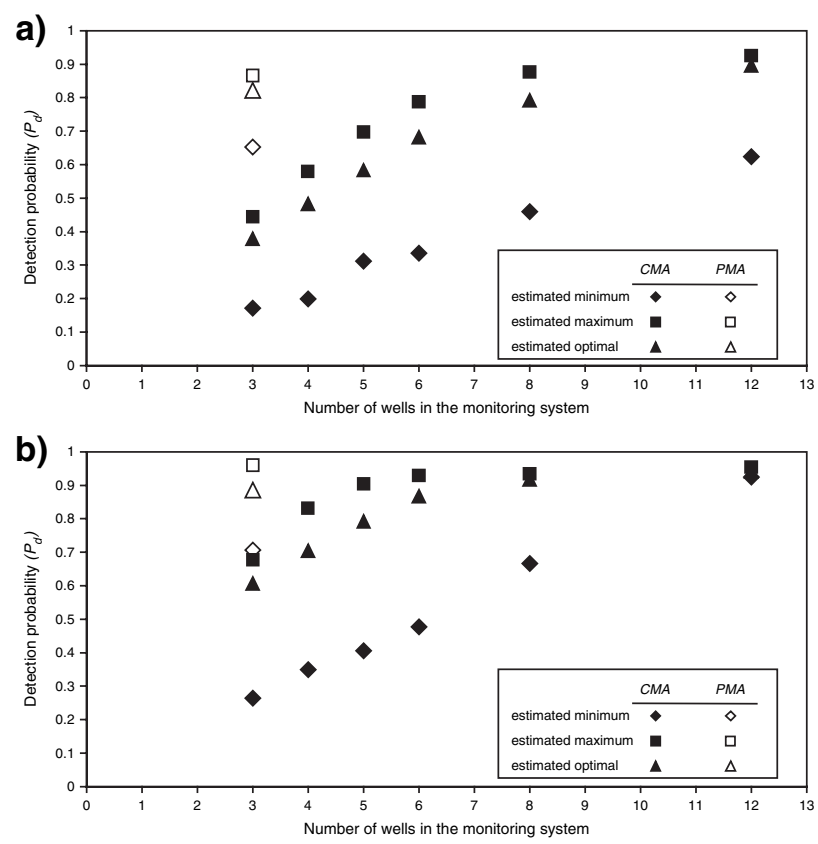

Figure 8. Comparison of the $C M A$ and the $P M A$ (pumping rate $=100 \mathrm{~L} / \mathrm{d}$ ) in terms of reliability in heterogeneous medium: (a) transverse dispersivity, $\alpha_{\mathrm{T}}=0.01 \mathrm{~m}$ and (b) transverse dispersivity, $\alpha_{\mathrm{T}}=0.03 \mathrm{~m}$. 

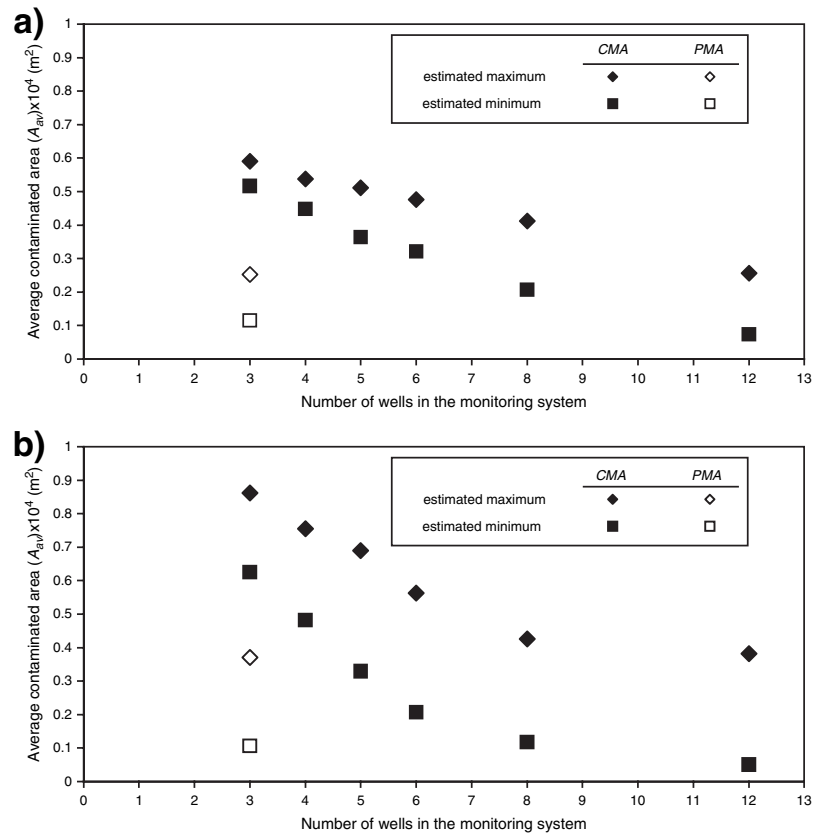

Figure 9. Comparison of the CMA and the PMA (pumping rate $=100 \mathrm{~L} / \mathrm{d}$ ) in terms of the average contaminated area in homogeneous medium: (a) transverse dispersivity, $\alpha_{\mathbf{T}}=$ $0.01 \mathrm{~m}$ and (b) transverse dispersivity, $\alpha_{\mathrm{T}}=0.03 \mathrm{~m}$.

area is reduced to $2330 \mathrm{~m}^{2}$. Thus, the PMA improves the efficiency of a widely used three-well monitoring system, by a factor of 1.88 and 2.72 in terms of maximum and minimum contaminated area, respectively. The values correspond to those that can be achieved by the monitoring systems composed of eight or more wells with regard to the CMA.
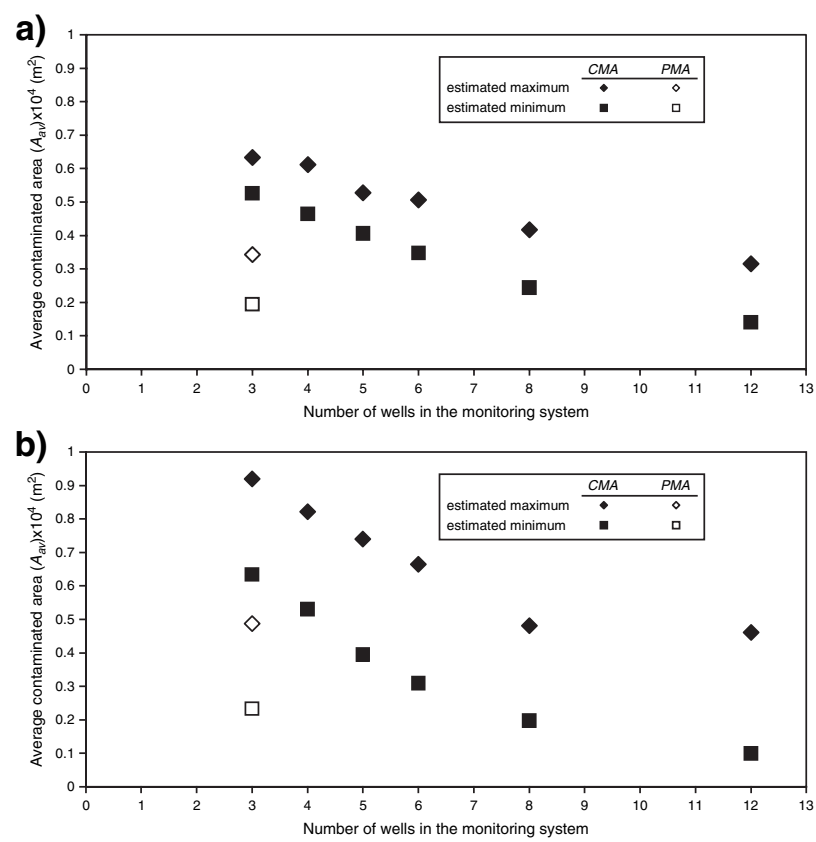

Figure 10. Comparison of the $C M A$ and the $P M A$ (pumping rate $=100 \mathrm{~L} / \mathrm{d}$ ) in terms of the average contaminated area in heterogeneous medium: (a) transverse dispersivity, $\alpha_{\mathrm{T}}=0.01$ $\mathrm{m}$ and (b) transverse dispersivity, $\alpha_{\mathrm{T}}=0.03 \mathrm{~m}$.
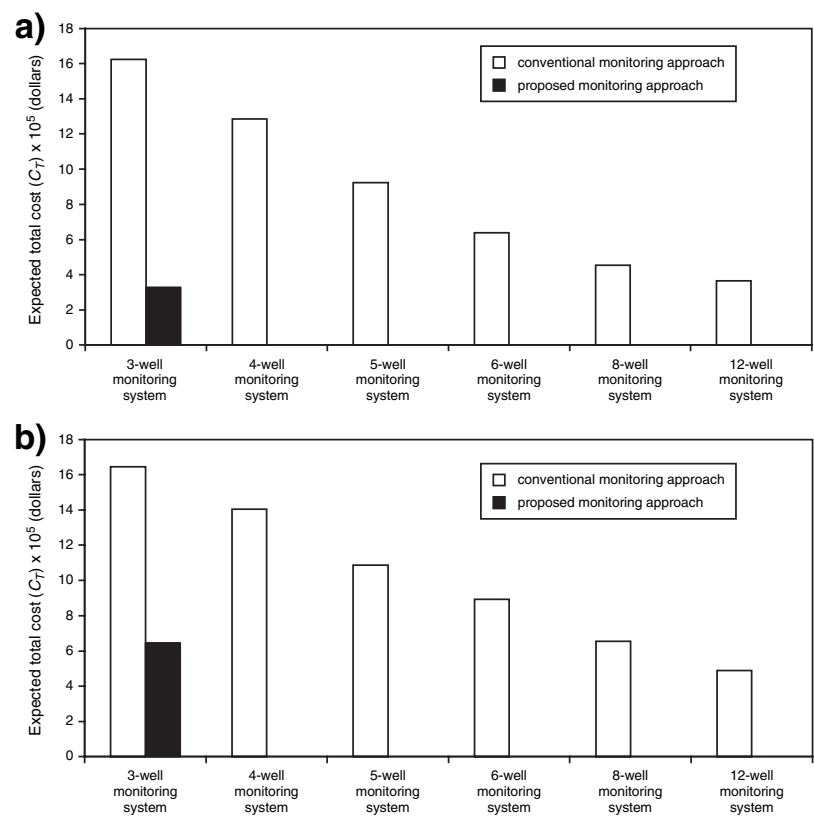

Figure 11. Expected cost as a function of number of wells in a monitoring system for transverse dispersivity, $\alpha_{\mathrm{T}}=0.03 \mathrm{~m}$ : (a) homogeneous medium and (b) heterogeneous medium.

\section{Cost Analysis}

Cost analysis has been carried out in order to estimate the improvement achieved by the PMA in terms of cost and to investigate its feasibility with respect to the CMA. Expected total cost, $C_{\mathrm{T}}$, is described as

$$
C_{\mathrm{T}}=C_{\mathrm{mw}} n_{\mathrm{mw}}+C_{\mathrm{r}} V_{\mathrm{av}}+C_{\mathrm{p}} Q_{\mathrm{p}} T_{\mathrm{m}}
$$

where $C_{\mathrm{mw}}$ is the unit installation and sampling cost of a monitoring well (\$/per well), $n_{\mathrm{mw}}$ is the number of wells in a monitoring system, $C_{\mathrm{r}}$ is the cost of remediation per unit volume $\left(\$ / \mathrm{L}^{3}\right), V_{\mathrm{av}}$ is the contaminated volume $\left(\mathrm{L}^{3}\right)$ to be cleaned up and is defined as $A_{\mathrm{av}}$ times the aquifer thickness, $C_{\mathrm{p}}$ is the unit cost for pumping $\left(\$ / \mathrm{L}^{3} / T\right), Q_{\mathrm{p}}$ is the pumping rate $\left(\mathrm{L}^{3} / T\right)$, and $T_{\mathrm{m}}$ is the total monitoring period $(T)$.

The unit installation and sampling cost is assumed to be fixed and $\$ 20,000$ per well. A unit remediation cost of $\$ 5 / \mathrm{m}^{3}$ and a unit pumping cost of $\$ 2 / \mathrm{m}^{3} / \mathrm{s}$ is taken in the cost estimation of monitoring systems (unit cost estimates are based on the study by James and Gorelick 1994). Figure 11 shows the expected cost values of optimal monitoring systems for both $C M A$ and $P M A$ in homogeneous and heterogeneous medium, where $\alpha_{\mathrm{T}}=0.03 \mathrm{~m}$. For $C M A$ the expected total cost decreases as the number of monitoring wells increases. However, compared with the $C M A$, the expected total cost of a three-well system reduces by a factor of 5 in homogeneous and by a factor of 2.5 in heterogeneous medium by pumping continuously from only one well, with a pumping rate of $100 \mathrm{~L} / \mathrm{d}$. Nevertheless, the unit remediation cost has a great influence on the difference in the expected cost values for both monitoring approaches. If the remediation of the contamination problem is easier than expected due to a cheaper unit cost of remediation, the reduction may be less. Conversely, if the remediation of the contamination problem is more 
difficult than expected, then the reduction in the cost will be even more since the unit cost of remediation will be higher.

\section{Conclusions}

Maximizing the likelihood of detecting contaminants and minimizing the contaminated area have been considered as design objectives. A simulation model is used to determine the optimal monitoring system where uncertainties due to subsurface heterogeneity and leak locations and the mentioned objectives are incorporated. A new monitoring approach is proposed to increase the efficiency of the widely used three-well monitoring system (minimum regulatory requirement) since, with the $C M A$, such a system more often is not adequate to accomplish the objectives of maximizing the detection probability while minimizing the contaminated area, particularly at lined landfills. This is mainly due to the limited capture zone of monitoring wells. Therefore, in the PMA, the main point is to increase the interception of contaminant plumes at early stages by broadening the capture zone of monitoring well(s) simply by continuous pumping from the monitoring well(s) with a small pumping rate.

Results from a detailed study of a hypothetical example showed that the new monitoring approach has improved the efficiency of an optimal three-well monitoring system by more than two times even under the least favorable circumstances, namely, in a heterogeneous highly dispersive $\left(\alpha_{\mathrm{L}}=0.3 \mathrm{~m}, \alpha_{\mathrm{T}}=0.03 \mathrm{~m}\right)$ medium. The same level of efficiency can be achieved for the CMA if a monitoring system is composed of more than eight wells. Dispersivity of the medium, heterogeneity, and the number of the wells are the important parameters that play a role in the efficiency of the monitoring systems. Furthermore, for the proposed new monitoring approach, pumping rate is an important factor that has influence on the reliability of the monitoring systems. However, the increase in the pumping rate will not make any further improvement once $100 \%$ reliability is achieved. On the other hand, analysis also showed that PMA is cost effective compared to CMA. However, one should keep in mind that although the minor changes would not influence expected cost values, still the unit remediation cost has a great influence on the difference in the expected cost values for both monitoring approaches. For instance, the easier the remediation of the contamination problem, the cheaper the unit cost of remediation, or vice versa.

\section{Acknowledgment}

The financial support by DIOC Water (Delft Interfacultair Onderzoeks Centrum voor de Duurzaam Gebouwde Omgeving) of the Delft University of Technology and Tauw for this research is gratefully acknowledged.

\section{References}

Ababou, R., D. McLaughlin, L.W. Gelhar, and A.F.B. Tompson. 1989. Numerical simulation of three-dimensional saturated flow in randomly heterogeneous porous media. Transport in Porous Media 4, no. 6: 549-565.
Bear, J. 1972. Dynamics of Fluid in Porous Media. New York: American Elsevier.

Bellin, A., P. Saladin, and A. Rinaldo. 1992. Simulation of dispersion in heterogeneous porous formations: Statistics, first-order theories, convergence of computations. Water Resources Research 28, no. 9: 2211-2227.

Elfeki, A.M.M. 1996. Stochastic characterization of geological heterogeneity and its impact on groundwater contaminant transport. Ph.D. diss., Delft University of Technology, Delft, The Netherlands.

European Community Council (ECC). 1999. Directive on the landfill of waste. Official Journal of the European Communities 31/EC, L182, 1-19.

Gelhar, L.W. 1986. Stochastic subsurface hydrology from theory to applications. Water Resources Research 22, no. 9: 135-145.

Hudak, P.F. 2002. Efficiency comparison of graphical approaches for designing contaminant detection networks in groundwater. Water Resources Research 38, no. 12: 18.1-18.5.

James, B.R., and S.M. Gorelick. 1994. When enough is enough: The worth of monitoring data in aquifer remediation design. Water Resources Research 30, no. 12: 3499-3513.

Kinzelbach, W. 1986. Groundwater Modeling: An Introduction with Sample Program in Basic. New York: Amsterdam Elsevier.

Mahar, P.S., and B. Datta. 1997. Optimal monitoring network and groundwater pollution source identification. Journal of Water Resources Planning and Management, ASCE, 23, no. 4: 199-207.

Massmann, J., and R.A. Freeze. 1987. Groundwater contamination from waste management sites: The interaction between risk-based engineering design and regulatory policy. 1. Methodology. Water Resources Research 23, no. 2: 351-367.

Meyer, P.D., A.J. Valocchi, and J.W. Eheart. 1994. Monitoring network design to provide initial detection of groundwater contamination. Water Resources Research 30, no. 9: 2647-2659.

Rouhani, S., and T.J. Hall. 1988. Geostatistical schemes for groundwater sampling. Journal of Hydrology 81, no. 1: 85-102.

Storck, P., J.W. Eheart, and A.J. Valocchi. 1997. A method for optimal location of monitoring wells for detection of groundwater contamination in three-dimensional heterogeneous aquifers. Water Resources Research 33, no. 9: 2081-2088.

U.S. EPA. 1986. RCRA Groundwater Monitoring Technical Enforcement Guidance Document. Washington, D.C.

Yenigül N.B., A.M.M. Elfeki, J.C. Gehrels, C. van den Akker, A.T. Hensbergen, and F.M. Dekking. 2005. Reliability assessment of groundwater monitoring networks at landfill sites. Journal of Hydrology 308, no. 1: 1-17.

\section{Biographical Sketches}

N. Buket Yenigül, MSc., corresponding author, is an Assistant Researcher with the Faculty of Civil Engineering and Geosciences, Water Resources Section, TU Delft, P.O. Box 5048, 2600 GA Delft, The Netherlands. Fax: (31) 15-2785915; n.b.yenigul@citg. tudelft.n.

Amro M.M. Elfeki, Ph.D., is an Associate Professor in the Department of Hydrology and Water Resources Management, Faculty of Meteorology, Environment and Arid Land Agriculture, King Abdulaziz University, P.O. Box 80208, Jeddah 21589, Kingdom of Saudi Arabia; aelfeki@kaau.edu.sa. On leave from Irrigation and Hydraulics Dept., Faculty of Engineering, Mansoura University, Mansoura, Egypt.

Cees van den Akker, Ph.D., is a Professor with the Faculty of Civil Engineering and Geosciences, Water Resources Section, TU Delft, P.O. Box 5048, 2600 GA Delft, The Netherlands. Fax: (31) 15-2785915; j.m.dejong@citg.tudelft.nl. 